\title{
The Arabidopsis AtRaptor genes are essential for post-embryonic plant growth
} Garrett H Anderson ${ }^{1}$, Bruce Veit ${ }^{2}$ and Maureen R Hanson*1

\author{
Address: ${ }^{1}$ Molecular Biology and Genetics, Cornell University, Ithaca, 14853, USA and ${ }^{2}$ AgResearch, Private Bag 11008 , Tennent Drive, Palmerston \\ North, 5301, New Zealand \\ Email: Garrett H Anderson - ganderson@salk.edu; Bruce Veit - bruce.veit@agresearch.co.nz; Maureen R Hanson* - mrh5@cornell.edu \\ * Corresponding author
}

Published: 21 April 2005

BMC Biology 2005, 3:12 doi:10.1186/1741-7007-3-12

This article is available from: http://www.biomedcentral.com/I741-7007/3/12

(c) 2005 Anderson et al; licensee BioMed Central Ltd.

This is an Open Access article distributed under the terms of the Creative Commons Attribution License (http://creativecommons.org/licenses/by/2.0), which permits unrestricted use, distribution, and reproduction in any medium, provided the original work is properly cited.
Received: 23 January 2005
Accepted: 2I April 2005

\begin{abstract}
Background: Flowering plant development is wholly reliant on growth from meristems, which contain totipotent cells that give rise to all post-embryonic organs in the plant. Plants are uniquely able to alter their development throughout their lifespan through the generation of new organs in response to external signals. To identify genes that regulate meristem-based growth, we considered homologues of Raptor proteins, which regulate cell growth in response to nutrients in yeast and metazoans as part of a signaling complex with the target of rapamycin (TOR) kinase.
\end{abstract}

Results: We identified AtRaptor IA and AtRaptor IB, two loci predicted to encode Raptor proteins in Arabidopsis. Disruption of AtRaptor I B yields plants with a wide range of developmental defects: roots are thick and grow slowly, leaf initiation and bolting are delayed and the shoot inflorescence shows reduced apical dominance. AtRaptor IA AtRaptor IB double mutants show normal embryonic development but are unable to maintain post-embryonic meristem-driven growth. AtRaptor transcripts accumulate in dividing and expanding cells and tissues.

Conclusion: The data implicate the TOR signaling pathway, a major regulator of cell growth in yeast and metazoans, in the maintenance of growth from the shoot apical meristem in plants. These results provide insights into the ways in which TOR/Raptor signaling has been adapted to regulate plant growth and development, and indicate that in plants, as in other eukaryotes, there is some Raptor-independent TOR activity.

\section{Background}

Plant development is remarkably plastic. Groups of totipotent cells termed meristems, which are maintained throughout the life of the plant, give rise to all postembryonic organs from roots and leaves to petals and fruit. This allows plants, unlike metazoans, to change their final body plans dramatically in response to environmental, hormonal and nutritional cues. While much has been learned about the determination of cell fates in the embryo [1] and the apical meristems [2-4], less is known about the genes that control the growth that occurs in cells emerging from the plant meristem.

TOR proteins were originally identified in budding yeast as the targets of rapamycin, a potent antibiotic that disrupts cell growth $[5,6]$. In both yeast and metazoans, TOR proteins mediate translation in response to nutrients [7]. Yeast TOR2 and mammalian mTOR also regulate cytoskeletal organization [8-13]. 
In both yeast and mammalian cells, TOR proteins form a complex, TORC1, with G $\beta \mathrm{L}[14]$ and Raptor (regulatory associated protein of TOR) [15-17]; their yeast homologues are LST8 and KOG1, respectively [17]. Raptor contains protein-binding domains, and the Raptor $\mathrm{N}$ terminus shows similarity to a caspase domain [18], though catalytic activity has yet to be shown. It has been reported that the strength of the TOR-Raptor interaction is regulated by nutrients, though there is not yet consensus on this point [15-17]. Raptor functions in TORC1 to recruit substrates for phosphorylation by TOR; Raptor binds TOR substrates S6kinase and eIF4E-BP, and is necessary for full TOR phosphorylation of these substrates in vitro $[16,19]$. The TOR-Raptor complex is thought to mediate the nutrient-sensitive regulation of cell growth; mutants in yeast lacking a functional complex cease cell growth in a manner that mimics rapamycin treatment [17]. A second TOR complex, TORC2, involves G $\beta \mathrm{L}$, Rictor (rapamycin-insensitive companion to TOR) and perhaps other proteins $[13,17,20]$. This complex is unaffected by rapamycin, and is thought to mediate TOR cytoskeletal regulation.

In Arabidopsis the single TOR homologue, AtTOR, is critical for plant development $[21,22]$. AtTOR insertion homozygote embryos undergo cell division but are unable to gain cell volume or undergo apical-basal differentiation. Although AtTOR transcripts accumulate in all tissues assayed [22], an AtTOR-Gus fusion transcript is only translated in meristematic tissue and the immediately adjacent expanding cells [21]. A TOR-specific microRNA conserved between Arabidopsis and rice [23] implicates post-transcriptional regulation in AtTOR expression. Rapamycin perturbs the activity of the nutrient-sensitive TOR complex in yeast and mammals. Arabidopsis, however, is insensitive to rapamycin, [21] precluding this approach to the elucidation of Arabidopsis TOR signaling.

Here we report a characterization of AtRaptor1A and AtRaptor1B, which encode the two Arabidopsis Raptor homologues. We found defects in both root and shoot growth in AtRaptor1B disruption lines, resulting in slow leaf initiation, late flowering and increased branching. We also found that AtRaptor1A AtRaptor1B double mutant homozygotes undergo normal seedling development, but exhibit only minimal post-embryonic meristem activity. We contrast these results with those found previously for the AtTOR mutant, and discuss the implications of this work for TOR signaling in plants.

\section{Results \\ Characterization of the Arabidopsis Raptor homologues}

To search for plant homologues of mammalian Raptor, we surveyed the completed Arabidopsis genome and found two loci that might encode proteins highly similar to Raptor at the north ends of chromosomes 3 and 5. We did not find an expressed sequence tag (EST) for the first locus (AtRaptor1A; At5g01770). However, we used the 5'-rapid amplification of CDNA ends (RACE) to establish that the locus is transcribed to an mRNA with a $5^{\prime}$ end consistent with the predicted open reading frame (ORF) of AtRaptor1A. The AtRaptor1A protein is predicted to be 1,346 amino acids in length. The second locus (AtRaptor1B; At3g08850) was represented by a single EST (accession no. AY769948). We used 5' RACE to confirm that the EST contained the full ORF of AtRaptor1B. The AtRaptor1B locus is transcribed to a $4.8 \mathrm{~kb}$ mRNA containing 23 exons, and the protein is predicted to be 1,344 amino acids in length. Predicted AtRaptor1A and AtRaptor1B proteins show $80 \%$ identity over their entirety.

To determine the time of divergence between the two AtRaptor loci, we searched for Raptor homologues in available plant genome data using AtRaptor1A and AtRaptor1B as query sequences. Full-length Raptor loci were discovered in the available rice (Oryza sativa subspecies japonica) and alfalfa (Medicago truncatula) genome sequences. Using AtRaptor $1 B$ and partial cDNA sequence (where available) as guides, we determined putative protein sequences from these loci. Alignment of these sequences with AtRaptor1A, AtRaptor1B, mammalian Raptor, the budding yeast Raptor homologue KOG1 and the fission yeast Raptor homologue Mip1p showed that there is a striking degree of conservation among all Raptor homologues (Fig. 1A; for the complete alignment [see additional file 1]). AtRaptor1B and the Saccharomyces cereviseae Raptor homologue KOG1, the most divergent member included in the analysis, show $28 \%$ identity throughout their length. All plant Raptor homologues encode the Raptor $\underline{N}$-terminal Conserved / Caspase (RNC/C) motif, HEAT repeats and WD40 motifs first identified in fission yeast Mip1 and characteristic of all Raptor proteins $[25,26]$ (Fig. 1B). From this alignment we generated a phylogeny of the Raptor homologues (Fig. 1C). The predicted AtRaptor proteins resolve to a single clade with a high degree of confidence, indicating that the duplication of the AtRaptor loci post-dates the divergence of Arabidopsis from Medicago, and that the loci are likely to encode functionally redundant proteins.

\section{Identification of knockout mutants in AtRaptor IA and AtRaptor IB}

To gain insight into the function of the AtRaptor proteins, we searched for insertion alleles of each locus among the publicly available sequenced T-DNA insertion lines. Insertion mutant lines SALK_043920 and SALK_078159, with disruptions to the AtRaptor1A and AtRaptor1B loci, were obtained from the Arabidopsis Biological Resource Center 


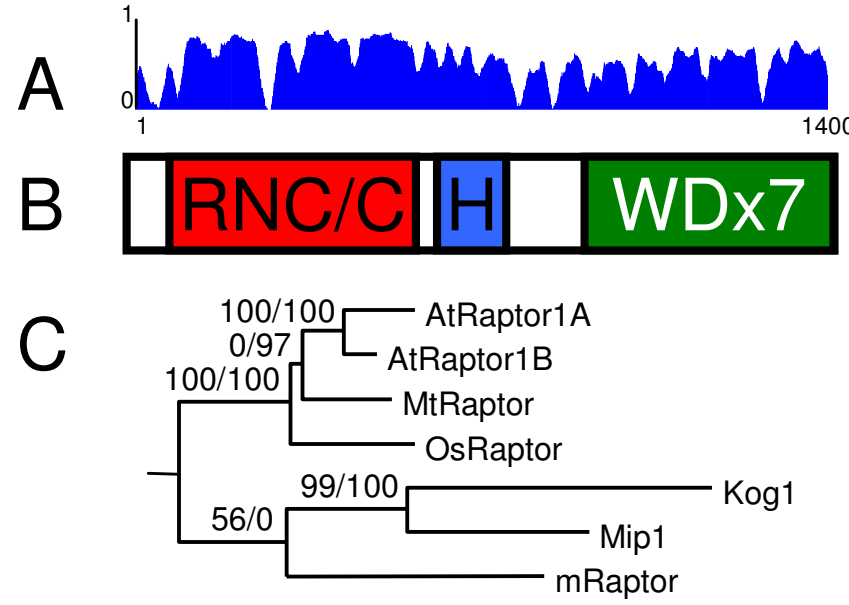

Figure I

Raptor proteins in eukaryotes are highly conserved. (A) Similarity plot of Raptor homologues from the vascular plants Arabidopsis, Medicago truncatula and Oryza sativa, the fungus $S$. pombe (Miplp), and mammals. The $X$-axis represents residue number; the $Y$-axis represents percent identity at that residue from $0 \%(0)$ to $100 \%$ (I). (B) Schematic diagram showing the position of the Raptor $\underline{N}$-terminal Conserved / putative Caspase domain (RNC/C) region, $\underline{\text { HEAT }}$ repeats $(H)$, and $\underline{W}-40$ repeats (WDX7) common to all Raptor proteins. (C) Phylogeny of plant, animal and fungal Raptor proteins. Bootstrap values, calculated using both parsimony (left) and maximum likelihood (right) are shown to the left of the clades they describe. The two Arabidopsis Raptor proteins, AtRaptor IA and AtRaptor IB, resolve as a single clade with $100 \%$ confidence. The alignment was generated using Megalign (DNAStar), the similarity plot was generated from this alignment using VectorNTI, and bootstrap values were calculated using PAUP*4.0b.

(USA). Lines homozygous for each insertion (referred to as $1 \mathrm{~A}-/$ - and $1 B-/-$, respectively) were identified via polymerase chain reaction (PCR), and RNA from floral buds of insertion homozygotes was used for reverse-transcriptase-PCR (RT-PCR) to assay for accumulation of wild-type transcripts from the disrupted locus. AtRaptor1A transcripts could not be detected in $1 \mathrm{~A}$-/- buds, but were detected in $1 B-/$ - buds. AtRaptor $1 B$ transcripts were not detected in $1 \mathrm{~B}-/$ - buds, but were detected in $1 \mathrm{~A} \mathrm{-/}$ - buds. Both transcripts were detected in wild-type Columbia (Col) buds (Fig. 2).

\section{B-I- seedling roots grow slowly}

As a first step toward characterizing these mutant lines, $\mathrm{Col}, 1 \mathrm{~A}-/$ - and $1 \mathrm{~B}-/$ - seedlings were germinated on culture medium. Col and $1 \mathrm{~A}-/$ - seedlings were phenotypically

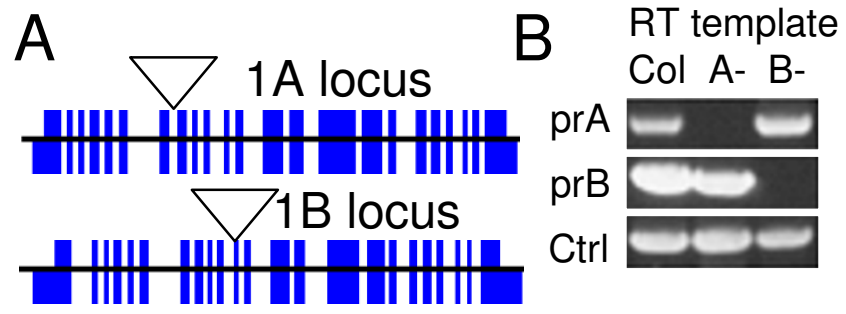

Figure 2

AtRaptor loci and insertion allele characterization. (A) AtRaptor IA and AtRaptor IB loci. Genomic sequence is depicted as a thin central line. Thick blocks indicate exons. Coding exons span the central line; exons encoding untranslated regions are fully below the central line. The positions of the T-DNA insertions are depicted with inverted triangles. (B) Reverse-Transcribed RNA-template Polymerase Chain Reactions (RT-PCR) on plants homozygous for both wildtype AtRaptor alleles (Col), the AtRaptor IA insertion allele (A) or the AtRaptor IB insertion allele (B-), using primers spanning the AtRaptor IA insertion site, the AtRaptor I B insertion site, or control primers. Both AtRaptor insertion alleles abolish accumulation of the wild-type transcript from their locus.

indistinguishable. However, B-/- seedlings showed distinct root growth defects: roots were thick, coiled and densely covered with hairs, and the roots had difficulty penetrating the medium (Fig. 3A). Plate-grown $1 B-/-$ seedlings were repeatedly shorter than $\mathrm{Col}$ or $1 \mathrm{~A}-/$ - seedlings. Although dark grown $1 \mathrm{~B}-/$ - seedlings were also shorter than Col or $1 \mathrm{~A}-/-$, the difference in size was much less (Fig. 3B). While dark-grown etiolated seedlings grow primarily through the expansion and division of embryonic hypocotyl cells, light-grown seedlings grow primarily through the production of cells from the root apical meristem. Thus the difference in size between Col, 1A-/- and $1 \mathrm{~B}$-/- seedlings results primarily from a reduction in root apical meristem growth rather than a general defect in cell expansion or metabolism.

The roots of $1 \mathrm{~B}$-/- seedlings grown on $90^{\circ}$ inclined plates were morphologically wild type but shorter than Col roots. When the plates were rotated to lay flat horizontally, new growth from the root apex of $1 B-/$ - seedlings produced thick, coiled roots (though the roots were not densely covered in hairs), while the previously produced root tissue remained morphologically wild type (Fig. 3C). Only those sections of the root not in contact with the medium were hairy (Fig. 3A). Thus the root growth disturbance was a defect in growth into agar rather than in gravitropism, though in the absence of resistance, roots still grew more slowly than wild type. 
A

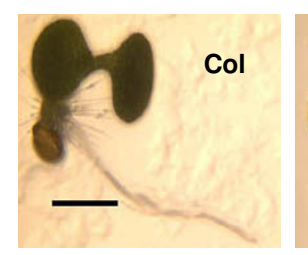

C

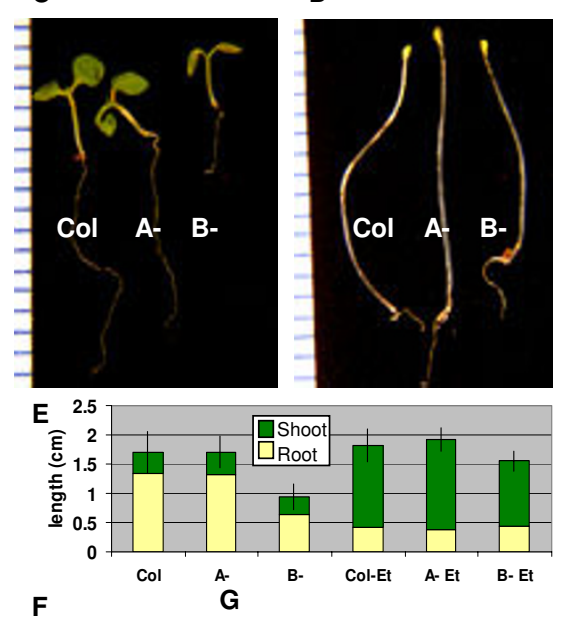

$\mathbf{F}$

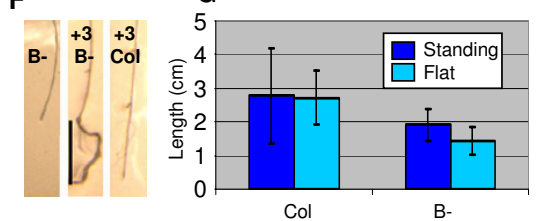

H

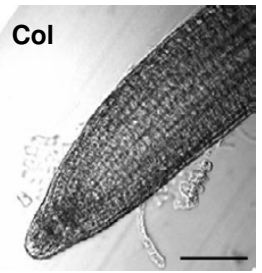

I

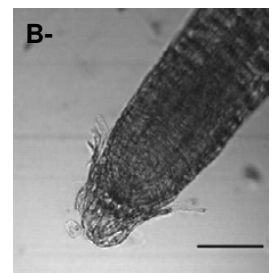

Figure 3

Seedling root phenotype of AtRaptor I B-/- (B-)

mutants. (A), (B). Col and B- seedlings on growth medium, four days after germination. The $B$ - root has not penetrated the medium and is thick, hairy and coiled. (C) Col, A- and Bseedlings at 8 days after germination in light. Scale bar is in $\mathrm{mm}$. (D) Same genotypes and age as C, grown in the dark. (E) Measurements of populations grown as in C, D. Root length is indicated in tan; shoot length is dark green. (F) B- and Col seedlings grown on vertical plates for 12 days, and then returned to horizontal growth for three days. B- roots are thin, straight and hairless on vertical plates (compare to $3 \mathrm{~B}$ ), and revert to coiled growth only in tissue generated after being placed horizontally. (G) Quantification of results in (F). $B$ - seedlings grown on vertical plates are intermediate in length between flat-grown B- seedlings and Col seedlings. $(\mathrm{H})$, (I) Col and B- root tips, viewed under bright field microscopy. Scale bar $=100 \mu \mathrm{m}$. B- root tips contain all the cell types seen in Col root tips, but the overall morphology is blunt and rounded compared to Col.
Col and 1B-/- root apical meristems (RAM) were examined using bright-field microscopy (Fig. 3I). 1B-/- seedling RAMs contained all cell types present in Col RAMs but their root morphology was much more blunt; this difference appeared to be localized to the $1 \mathrm{~B}-/$ - zone of elongation. $1 B$-/- root tips showed a tendency to shed their root caps, although shedding was sometimes observed in Col root tips as well.

\section{B-l- shoots show developmental and morphological defects}

To measure developmental defects resulting from AtRaptor insertions, we grew Col, $1 \mathrm{~A}-/-$ and $1 \mathrm{~B}-/$ - lines on soil, and scored their rates of leaf initiation, time to floral bud initiation, rate of cauline leaf initiation, time to flowering and number of floral shoot apices. $1 \mathrm{~B}-/$ - plants were smaller, had a slower rate of leaf initiation, and bolted and flowered later than did Col or 1A-/- plants (Fig. 4AC).

Mature 1B-/- plants were conspicuously bushier than Col or 1A-/- homozygotes (Fig. 5). The primary shoot apex was shorter than that of Col plants and ended prematurely in a terminal inflorescence of infertile flowers. The growth of the plant was then taken over by axillary shoots and by secondary shoots from the basal rosette.

To quantify this phenotype, Col and $1 \mathrm{~B}-/$ - lines were grown to maturity in 16-hr days, and shoot architecture for an individual plant was scored at the shattering of the first silique. $1 \mathrm{~B}-/$ - plants showed reduced shoot height, reduced primary stem length, increased axillary branch number and an increased number of secondary shoots compared to Col and 1A-/- plants (Fig. 5B, E). 1B-/branch numbers, though variable, were significantly larger than those of wild type or $1 \mathrm{~A}-/$-. Student's $t$-Tests of sample pairs assuming equal variance yielded P-values of 0.0027 and 0.0004 for $1 B-/-v s$. Col and $1 B-/-v s .1 A-/-$ axillary branch number, respectively, and 0.0026 and 0.0013 for $1 \mathrm{~B}-/-v s$. Col and $1 \mathrm{~B}-/-v \mathrm{~s}$. 1A-/- secondary shoot number. Axillary branch length did not differ significantly from Col values (Fig. 5F). This phenotype became more pronounced later in plant development and was more conspicuous under short days.

\section{Complementation of the AtRaptor I B-/- phenotype}

To confirm that the collection of phenotypes observed in the AtRaptor1B-/- homozygotes was due to the mutant 1B allele, 1B-/- plants were transformed with a construct containing the AtRaptor $1 B$ ORF and 5' UTR and driven by the AtRaptor1B promoter. Some of these transformants showed wild-type transition to bolting, shoot branching and root growth (data not shown). We conclude that the $1 B$-/- phenotypes described above result from homozygosity for the lesion at the AtRaptor1B locus. 


\section{AtRaptor transcripts accumulate in dividing and expanding cell tissues}

A combination of in situ RNA hybridization and in silico expression analysis was used to determine the RNA accumulation pattern of AtRaptor transcripts in Arabidopsis. The AtRaptor1B cDNA sequence was used to generate an AtRaptor probe to assay transcript accumulation in the shoot tips of wild-type plants. Since AtRaptor1A and AtRaptor $1 B$ show $80 \%$ identity through the length of their transcripts, this probe likely detected expression of both loci.AtRaptor transcripts were detected throughout the shoot tip, in all organs of the differentiating floral bud, and deep into the differentiated inflorescence stem (Fig. 6A). Signal intensity faded with the distance from the shoot apex. This accumulation pattern differed from that of actin (Fig. 6B), which was more prominent in dividing cells of the apex. Notably, AtRaptor accumulation is not restricted to the primary shoot apex.

To obtain an estimate of the relative levels of AtRaptor1A and AtRaptor $1 B$ in the signal seen in Fig. 6A, AtRaptor1A and AtRaptor $1 B$ locus IDs were used to query the Genevestigator Arabidopsis thaliana Microarray Database and Analysis Toolbox [27]. AtRaptor1A and AtRaptor1B accumulate in all developmental stages (Fig. 6C). However, AtRaptor1B accumulation levels are consistently fourfold higher than those of AtRaptor1A. A second analysis of accumulation by tissue rather than developmental stage produced similar results. We conclude that AtRaptor1A and AtRaptor $1 B$ show similar expression patterns.

\section{A-/- IB-/- double homozygote mutant seedlings show minimal meristem growth}

The high degree of similarity between AtRaptor1A and AtRaptor1B led us to suspect that they may be at least partially functionally redundant. To eliminate all AtRaptor activity in a single line, $1 \mathrm{~A}$-/- and $1 \mathrm{~B}$-/- lines were crossed. No $1 A-/-1 B$-/- plants could be identified among the $F_{2}$ of this cross on soil. Phenotypically wild-type $1 \mathrm{~A}-/-1 \mathrm{~B}+/-$ plants were identified via PCR using the primer pairs described in Materials and Methods and their progeny (205 seedlings) were examined on agar plates. Of these, 165 seedlings ( $80 \%)$ appeared wild type (Fig. 7A). It was determined by PCR that these seedlings carried a wildtype $1 B$ allele (32/32 tested). The remaining seedlings $(40 / 205 ; 20 \%)$ germinated but showed minimal postembryonic shoot meristem growth (Fig. 7A). It was determined by PCR that these seedlings were genetically $1 \mathrm{~A}-/$ $1 B-/-$ (32/32 tested). This genotyping confirmed that the seedling arrest mutant phenotype co-segregated with homozygosity for the AtRaptor1B mutant allele in the AtRaptor1A mutant background. We concluded that this seedling phenotype corresponded to the $1 \mathrm{~A}-/-1 \mathrm{~B}-/-$ double mutant.
A

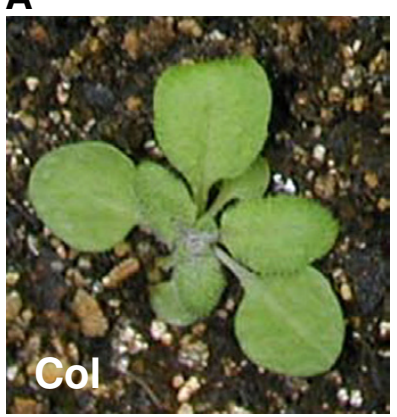

B

C

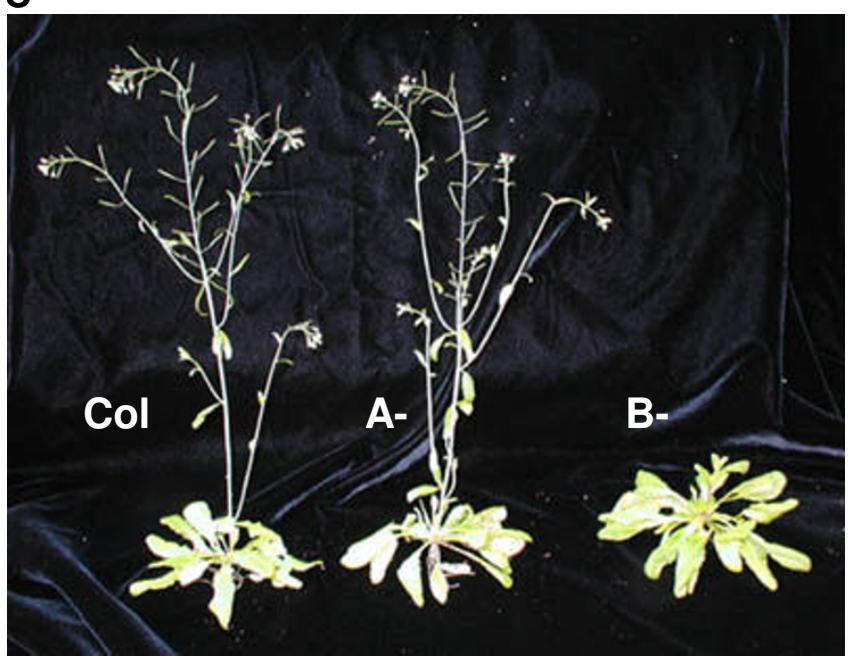

D

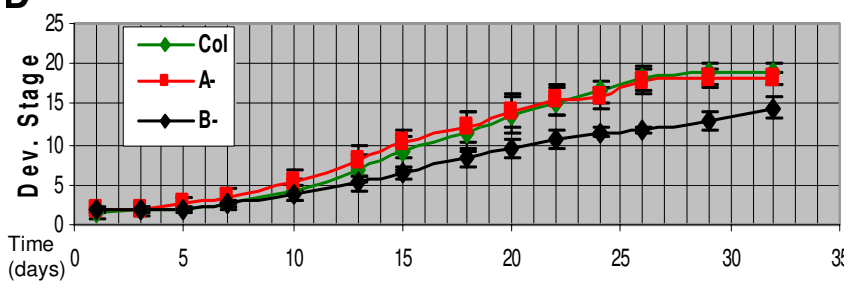

Figure 4

AtRaptor IB-/- plants grow slowly. (A), (B) Col and Bplants at I5 days after germination on soil. (C) B- plants bolt later than Col or A-. Shown are shoots from plants I month after germination. (D) Growth curve of Col, A- and B- plants. The $X$-axis represents time after production of the first leaf. The $\mathrm{Y}$-axis represents the number of rosette leaves up to II; presence of a floral bud is 12; number of cauline leaves plus 12 is $13-16$, and values above 16 are the number of shoot apices harboring flowers plus I5. B- plants show slower leaf initiation, later bolting (though at a similar rosette leaf number as Col and A-) and later flowering. 

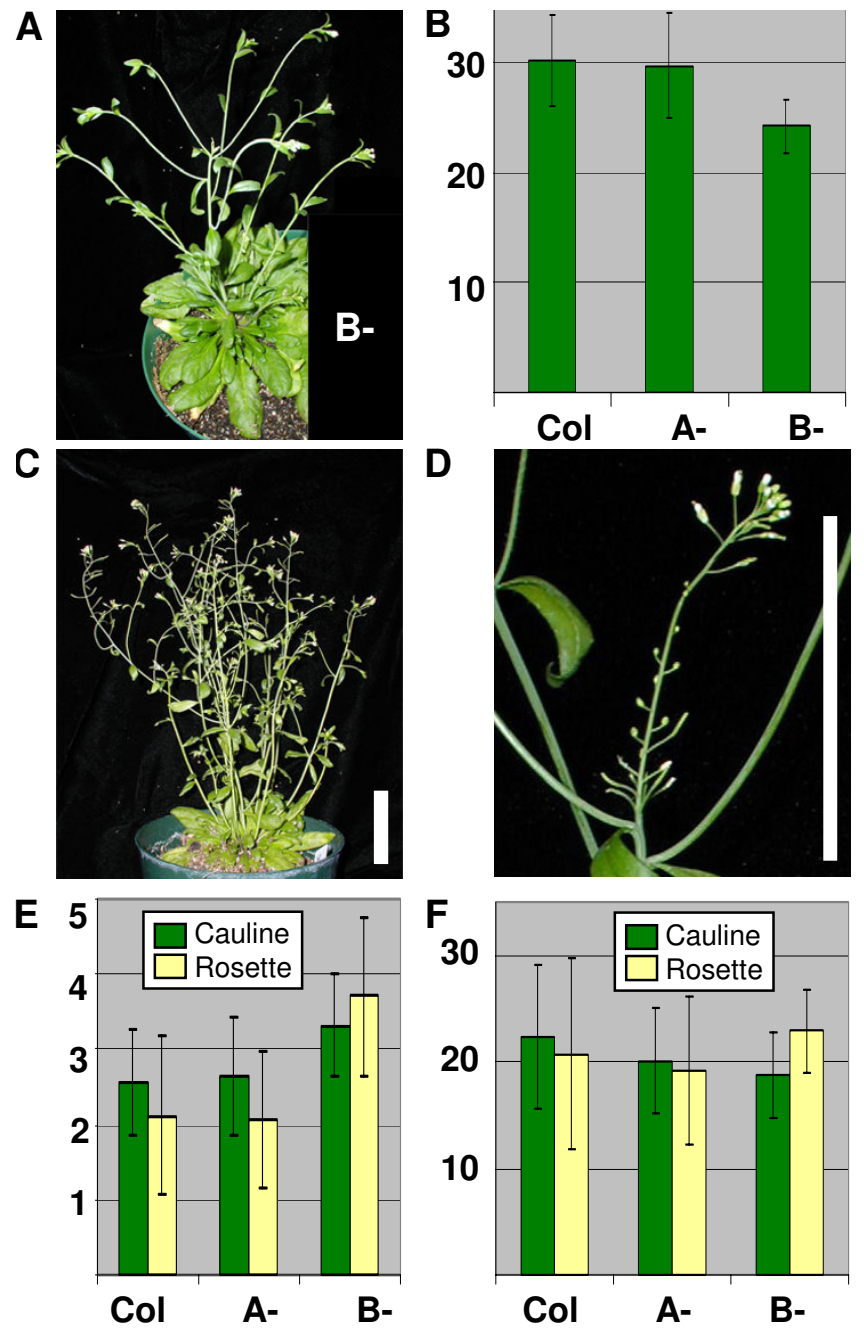

Figure 5

AtRaptorIB-/- plants show altered shoot architecture. (A) B- plant at flowering. The primary shoot apex, center, has ceased growth and is surpassed by axillary branches. Compare to Col, A- in 3C. (B) Col, A- and B- primary shoot length. (C) Mature B- plant, showing a bushy phenotype due to decreased primary shoot growth and increased branching. (D) Shoot apex of the plant in C, magnified 4.5×. Axillary branches outgrow the primary shoot, which arrests in a whorl of sterile flowers. (E) Col, A- and B-cauline and rosette branch number. (F) Col, A- and B-cauline and rosette branch length. $B$ - primary shoots are smaller than Col or A-, and secondary shoots initiate more frequently than Col or A- but are not significantly longer than Col or A-

We examined $1 \mathrm{~A}-/-1 \mathrm{~B}-/$ - double mutant seedling roots via bright field microscopy to ascertain the extent of their post-embryonic growth defect. $1 \mathrm{~A}-/-1 \mathrm{~B}-/$ - roots were conspicuously narrower than wild type. The columella, quiescent center, vasculature, pericycle, endodermis and

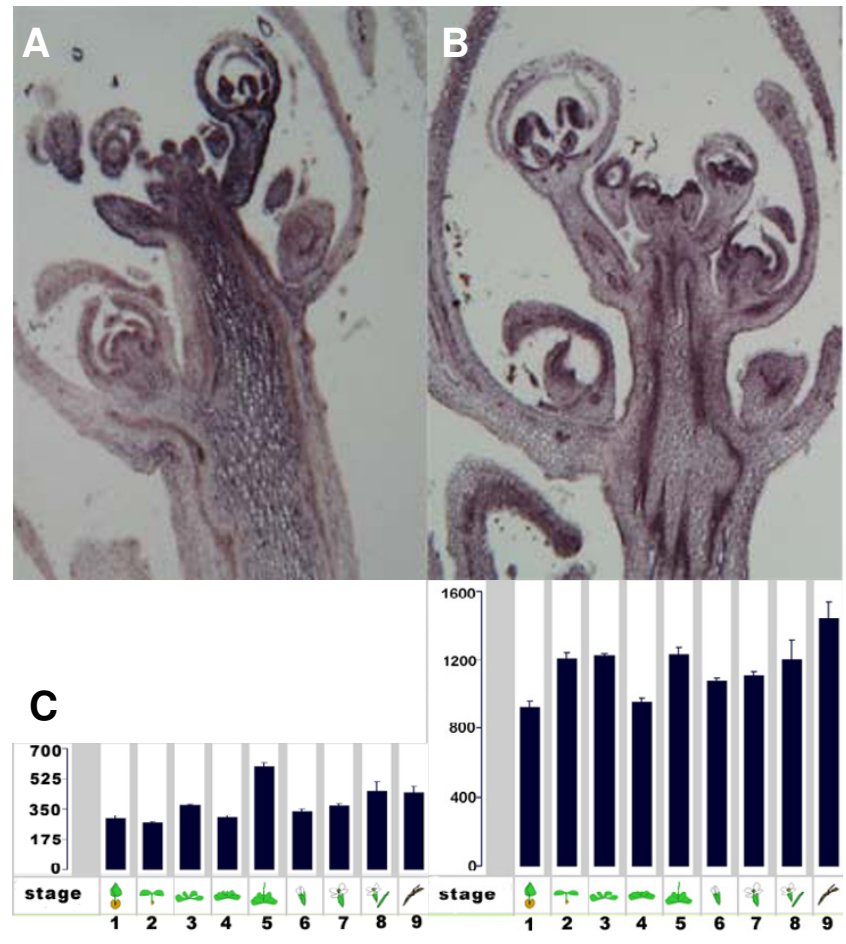

\section{Figure 6}

AtRaptor accumulation pattern. (A) AtRaptor transcripts accumulate throughout the floral shoot apex, stem and differentiating floral buds. Accumulation is not confined to dividing or meristematic cells, but fades in intensity away from the apex. (B) Adjacent tissue slice, probed with actin. AtRaptor and actin transcript accumulation patterns differ. (C) In silico analysis of AtRaptor IA (left) and AtRaptor IB (right) accumulation from 1434 developmental gene chip experiments. Results are given by developmental stage (X-axis) and in terms of gene chip-normalized expression levels ( $Y$-axis). Expression levels are shown to scale. Developmental stages are as follows: I, I.0-5.9 days; $2,6.0-13.9$ days; 3, 14.0-17.9 days; 4 , 18.0-20.9 days; 5 , 21.0-24.9 days; $6,25.0-28.9$ days; 7, 29.0-35.9 days; 8, 36.0-44.9 days; 9, 45.0-50.0 days. Analyses performed via the genevestigator website https:// www.genevestigator.ethz.ch.

cortex are present though all smaller than wild type; the outer layer of cells is difficult to identify. Further up the root, root hairs were clearly visible, indicating that the RAM had produced some mature root cell types (Fig. 7D, E).

To gain a better understanding of the double mutant shoot apical meristem (SAM) defect, Col and 1A-/- 1B-/7-day seedlings were fixed and coated, and their shoot apexes were observed with a scanning electron microscope. In Col seedlings, leaves 1 and 2, with trichomes, 

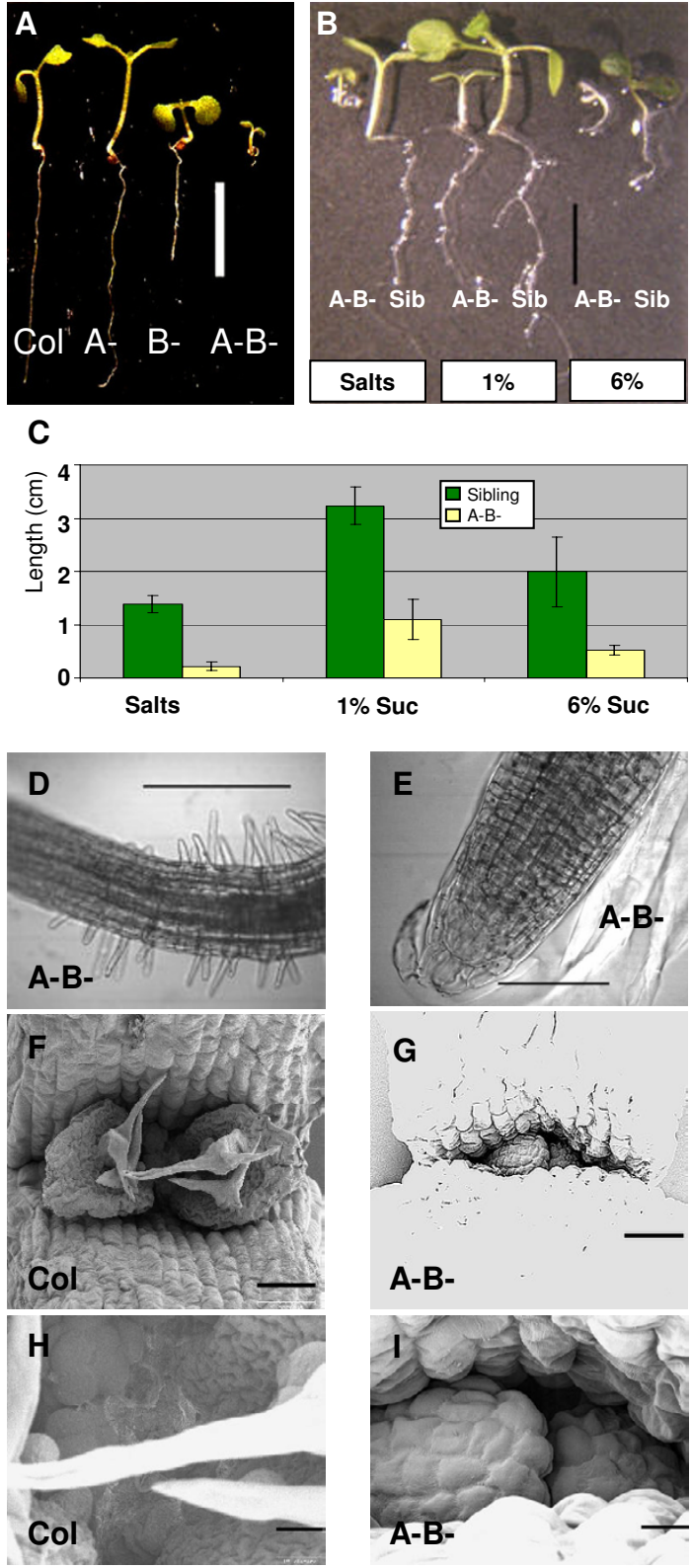

Figure 7

AtRaptorIA-/- IB-/- double mutants. (A) Col, A-, B- and $A-B$ - seedlings at seven days on growth medium with no sucrose. (B) IA-/- IB+/- progeny germinated on growth medium supplemented with $0 \%, 1 \%$ or $6 \%$ sucrose. Shown for each treatment is an A-B- seedling and an A-/- B+ sibling. $A-B-$ seedlings on $1 \%$ sucrose show significant root growth and minimal leaf buds. Scale bar for $A, B=5 \mathrm{~mm}$. (C) Quantification of results in (B). (D) A-B- root tips grown on $1 \%$ sucrose lack an epidermal cell layer. (E) A-B- roots form root hairs on I\% sucrose. Scale bar $=100 \mu \mathrm{m}$. Compare to $3 \mathrm{H}$, I. $(F, G, H, I)$ Scanning electron microscopy on Col, A-B- shoot apices from growth medium plates. As in (B), A-B- seedlings show minimal (SAM) activity. Primordia for leaves $I$ and 2 form but do not expand significantly. Scale bar $=50 \mu \mathrm{m}(\mathrm{F}$, $\mathrm{G})$ or $\mathrm{I} 5 \mu \mathrm{m}(\mathrm{H}, \mathrm{I})$. were clearly visible; primordia for leaves 3,4 and 5 were visible under higher magnification. 1A-/- 1B-/- shoot apexes showed minimal SAM activity: leaves 1 and 2 were produced, but were smaller than wild type and did not separate to reveal any further leaf primordia (Fig. 7F, G, H, I).

In order to determine whether a plant hormone or signaling molecule could restore post-embryonic growth to these arrested seedlings, progeny of $1 \mathrm{~A}-/-1 \mathrm{~B}+/$ - individuals were germinated on plates containing a variety of plant hormones and signaling molecules and scored for their total seedling lengths at 10 days post germination. Signaling molecules tested included the gibberellin GA3 (100 $\mathrm{nM}$ to $10 \mu \mathrm{M})$, abscisic acid ( $100 \mathrm{nM}$ to $10 \mu \mathrm{M})$, the auxin 2,4-D ( $1 \mathrm{nM}$ to $10 \mu \mathrm{M})$, the ethylene precursor ACC $(1 \mu \mathrm{M}$ and $10 \mu \mathrm{M})$, sucrose $(1 \%)$ and glucose $(6 \%)$. We also attempted dark treatment. None of these treatments restored wild-type SAM growth to the quarter of the progeny showing seedling arrest. $1 \mathrm{~A}-/ \mathrm{-}-1 \mathrm{~B}-/$ - seedlings did show a significant response to sucrose (Fig. 7B, C). Addition of $1 \%$ sucrose, which is a growth signaling factor as well as a carbon source [28], to the growth medium promoted growth in Col, $1 \mathrm{~A}-/-, 1 \mathrm{~B}-/-$ and $1 \mathrm{~A}-/-1 \mathrm{~B}-/-$. In sibling seedlings, the addition of sucrose elicited a twofold increase in seedling length; in the double mutants the increase was five-fold. Germination on $6 \%$ sucrose, which signals growth arrest in Col plants [28], yielded sibling plants $1-1.5 \times$ the size of seedlings grown on $1 / 2$ Murashige \& Skoog (MS) salts with no sucrose added. 1A/- $1 B$-/- seedlings on $6 \%$ sucrose were $2.3 \times$ the length of those grown on $1 / 2 \mathrm{MS}$ salts. A similar result was observed among $1 \mathrm{~A}-/-1 \mathrm{~B}+/$ - progeny germinated in the dark on $1 /$ 2 MS salts plates, and the length of $1 \mathrm{~A}-/-1 \mathrm{~B}-/$ - seedlings was increased more than $3 \times$ under these conditions. The absolute length of the double mutant seedlings was in all cases still substantially smaller that that of sibling seedlings for a given treatment, and the increase in length was largely due to hypocotyl elongation or root growth. Minimal SAM activity was observed.

\section{Discussion}

Axillary branch growth in Arabidopsis and other vascular plants is governed indirectly by auxin produced at the primary shoot apex, which acts through an undetermined secondary messenger to repress axillary meristem growth [29]. The shoot morphology of $1 \mathrm{~B}-/$ - plants indicates a defect in primary SAM maintenance. The primary shoot is shorter than wild type and ends in a whorl of sterile flowers. The number and length of axillary shoots, both cauline and emerging from the basal rosette, is significantly increased. Notably, axillary meristems are quite viable, producing branches as long as or longer than wild type. The viability of axillary shoot apices points to a failure to maintain primary SAM activity, and a subsequent 
failure to repress axillary meristem activity. This defect is not shared by the axillary SAMs, which match or surpass the activity of their wild-type counterparts. A similar phenomenon is seen in erecta mutants [30] and may be quite common. The increased branching in AtRaptor1B mutant plants may result directly from reduced auxin production at the shoot apex or from a change in the auxin/cytokinin ratio. However, $1 \mathrm{~B}-/$ - mutants did not show defects in their ability to sense exogenous auxin (data not shown).

The endogenous AtRaptor1A locus cannot fully complement the disruption of AtRaptor1B despite the fact that their predicted proteins are $80 \%$ identical, share all conserved Raptor motifs and strongly resolve to a single clade in phylogenetic analysis. In silico analysis suggests that the failure of AtRaptor1A to mask AtRaptor1B lesions may be due to lower levels of AtRaptor1A expression globally rather than limited tissue-specific accumulation of AtRaptor1A. We did not assay for the complementation of AtRaptor1B lesions by transforming AtRaptor1B-/- plants with an AtRaptor1A construct. In contrast, AtRaptor1B can fully complement the loss of AtRaptor1A expression; indeed, a single wild-type AtRaptor $1 \mathrm{~B}$ allele in $1 \mathrm{~A}-/-1 \mathrm{~B}+/-$ plants is sufficient for wild-type growth.

Our ability to isolate AtRaptor1B mutant homozygotes conflicts with a recent report [24]. In that report, mutants homozygous for AtRaptor1B disruptions were lethal at or before the first asymmetric embryonic division, which is even earlier than the lethality of AtTOR mutant homozygotes. We are uncertain of the reason for the discrepancy in these results. We are able to recover $1 \mathrm{~B}$-/- plants, but Deprost et al. [24], working with the same AtRaptor1B allele (SALK_043920), described them as embryo-lethal. One possible explanation might be a variation in growth conditions, which could lead to a higher degree of stress on developing embryos in the experiments by Deprost et al. The fact that these authors observed some embryo arrest in wild type control lines supports this possibility. Disruption of AtRaptor1A yielded no significant phenotype in either our growth conditions or those of the previous report [24].

Simultaneous disruption of both AtRaptor loci resulted in growth-arrested seedlings, which undergo normal embryonic organogenesis but are unable to maintain post-embryonic growth from their SAMs. AtRaptor1A-/AtRaptor1B-/- double mutants' SAMs cease activity after the production of a few leaf primordia. We conclude that AtRaptor activity is essential for the maintenance of SAM activity, but not for SAM assembly or for the initiation of SAM activity.

Our results indicate that AtRaptor activity is not essential for embryonic organogenesis. In contrast, AtTOR-/- mutants arrest development early in embryogenesis [21]. The disparity between the AtRaptor1A-/- 1B-/- and the AtTOR-/-phenotypes indicates that AtTOR activity in embryonic development does not require AtRaptor. Thus if AtTOR is acting in a complex in embryonic development, this complex does not require AtRaptor.

In both yeast and mammalian cells, TOR functions in two distinct complexes (Fig. 8A). The first of these, TORC1, involves Raptor and regulates cell growth and translation in response to nutrients [14-17]. The second of these complexes, TORC2, regulates cytoskeletal organization and does not involve Raptor $[13,17,20]$.

AtRaptor-independent AtTOR activity in the plant embryo is consistent with the existence of two AtTOR complexes in Arabidopsis: 1) a Raptor-independent complex critical for early embryogenesis (and perhaps all stages of plant development), and 2) a Raptor-dependent complex that is dispensable for embryonic development but which is necessary for post-embryonic, meristem-driven plant growth (Fig. 8B).

\section{Conclusion}

By identifying the phenotypes resulting from partial and total disruption of AtRaptor activity, we have generated valuable tools for the study of plant TOR signaling. When viewed in the context of previous work on AtTOR [21,22], our work provides evidence for Raptor-independent TOR activity in land plant embryonic development - development that, notably, occurs in an environment made nutritionally and environmentally stable via maternal input to growth. We propose that in plants as in other eukaryotes there are two (or more) TOR complexes, only one of which involves Raptor. We further propose that the plant homologue of TORC1, involving TOR and Raptor, has been co-opted in plants from its ancestral role in nutrient sensing and cell growth to regulate the highly plastic postembryonic growth driven by the plant shoot apical meristem.

\section{Methods \\ Insertion lines}

The mutant lines SALK_043920 and SALK_078159, in which Agrobacterium-mediated T-DNA insertions disrupted the AtRaptor1A and AtRaptor1B loci in the Columbia (Col) genetic background, were generated by Joseph R. Ecker and the Salk Institute Genomic Analysis Laboratory (USA) and distributed by the Arabidopsis Biological Resource Center (USA) [31]. Lines were genotyped using the following primers: 1 Asm5 5'aaaaagtctcttagatgtagtttcagatg $3^{\prime}$ and 1 Asm $35^{\prime}$ attcagaatatacaatccaagcattagt $3^{\prime}$ to identify the AtRaptor1A wild-type locus; 1 Bsm5 5' ctgaccataacattctcttgtaggtaagg $3^{\prime}$ and $1 \mathrm{Bsm} 35^{\prime}$ aggcctgaactctaatgaacaaactctcc 3 ' to identify the AtRaptor $1 B$ wild-type locus; 

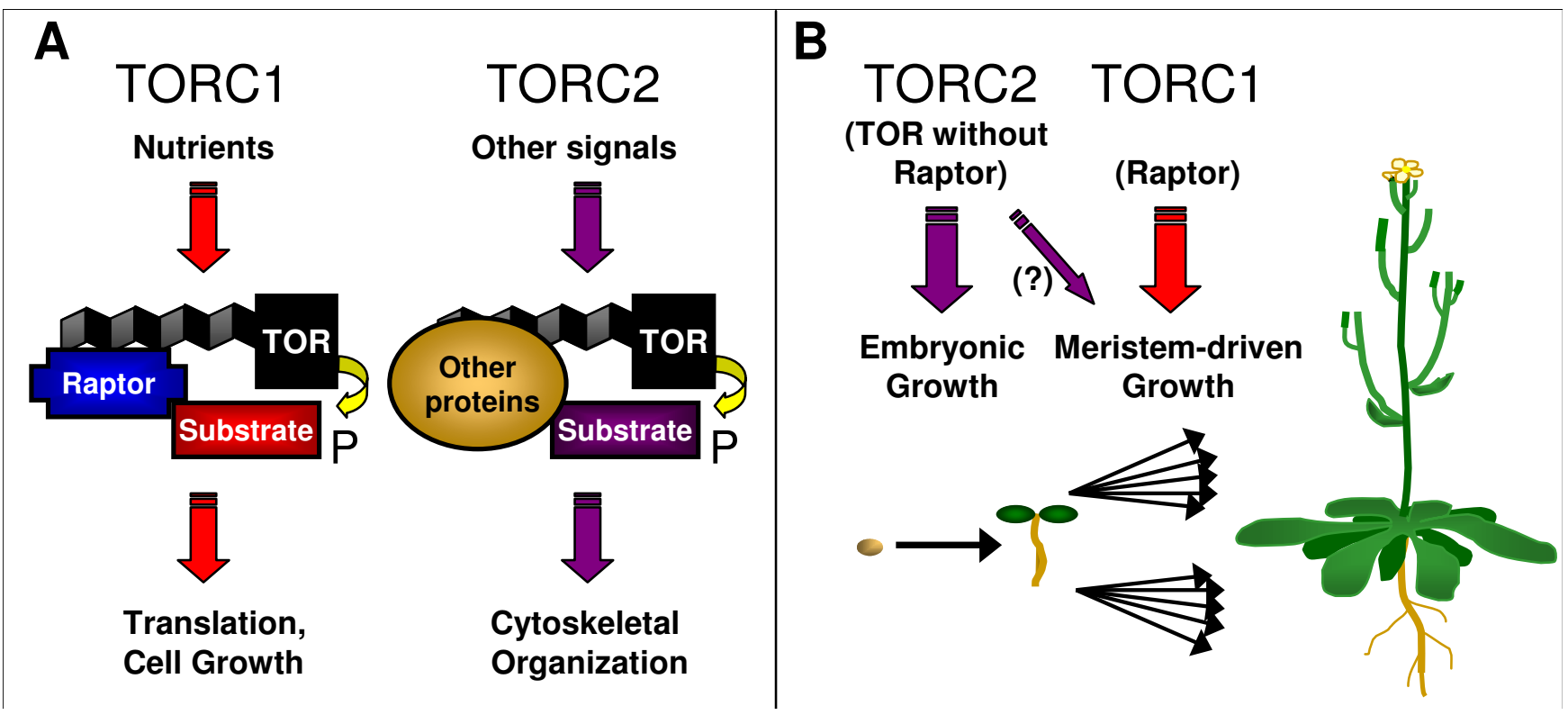

Figure 8

TOR functions in two complexes in eukaryotes. (A) TOR participates in two complexes in yeast and mammals. The first of these, TORCI, regulates cell growth in response to nutrient and hormonal signals. Raptor is integral for TORCI activity. The second of these, TORC2, regulates cytoskeletal organization. Its activity is nutrient-independent, and Raptor is not a component of TORC2. (B) Model of TOR function in plants. Embryonic development is indicated by the single horizontal arrow from zygote to seedling; meristem-driven post-embryonic development is indicated by the arrows emanating from the seedling root and shoot apices. TOR, acting independent of Raptor in a putative complex homologous to yeast and mammalian TORC2, is essential for embryonic development. TOR via TORC2 may play a role in post-embryonic development as well; the embryonic lethal AtTOR knockout phenotype precludes a definitive answer on this point. Raptor activity, in a putative plant homologue of TORCI, is dispensable for embryonic development but is essential for meristem-driven post-embryonic growth.

and 1 Bsm 5 or 1 Asm 5 and pROK-737 ( $5^{\prime}$ gggaattcactggccgtcgttttacaa $3^{\prime}$ ) to identify the respective AtRaptor mutant alleles. Mutant lines were crossed to Col wild type plants; the AtRaptor1B mutant phenotype cosegregated with the $1 \mathrm{~B}$ insertion allele in the $\mathrm{F}_{2}$ population.

\section{Growth conditions}

Plants were grown in a greenhouse with supplemental light to 16 hrs, with temperatures held at $22^{\circ} \mathrm{C}$ days and $17^{\circ} \mathrm{C}$ nights. Seeds for plate-grown seedlings were surface sterilized with $20 \%$ Chlorox, washed in $\mathrm{H}_{2} \mathrm{O}$, stratified for four days at $4^{\circ} \mathrm{C}$ and grown on $1 / 2 \times \mathrm{MS}$ salts, $0.3 \%$ Phytagel under $12 \mathrm{hr}$. daylight cycles.

\section{RT-PCR}

Buds from Col and insertion line homozygotes were snapfrozen in liquid $\mathrm{N}_{2}$. Total RNA was extracted from $0.3 \mathrm{~g}$ of tissue using TRIzol Reagent (Invitrogen) according to manufacturer's instructions. Resuspended RNA was thrice treated with DNA-free ${ }^{\mathrm{TM}}$ DNase treatment (Ambion). RNA was quantified with a spectrophotometer. cDNA was generated from $2 \mu \mathrm{g}$ of total RNA using Omniscript Reverse Transcriptase (Qiagen) and a poly- $\mathrm{dT}_{18}$ primer. PCR was performed using ExTaq polymerase (Takara) in a PTC-100 thermocycler. Primer sequences were as follows: $1 \mathrm{~A}+1828,5^{\prime}$ gctgcgtttatttggctgttattgtc $3^{\prime}$ and 1A-2800, 5' ctaggccagccagaggagtgtgagatg $3^{\prime} ; 1 \mathrm{~B}+1379, \quad 5^{\prime}$ aggccggcaaaacgatcgtaagacatt $3^{\prime}$ and $1 \mathrm{~B}-2774,5^{\prime}$ catcagcccagaggagccaagagg $3^{\prime}$. PCR cycling parameters were $95^{\circ} \mathrm{C}$ for 5 min followed by 35 cycles of $94^{\circ} \mathrm{C}$ for $30 \mathrm{sec}, 62^{\circ} \mathrm{C}$ for 30 sec, and $72^{\circ} \mathrm{C}$ for $1 \mathrm{~min}$.

\section{AtRaptor IB cDNA clone}

EST clone RZL03b06 corresponding to the 5' end of AtRaptor1B was ordered from Kazusa DNA Research Institute and sequenced (Accession number AY769948). RNA ligase-mediated RACE was performed on total RNA extracted from bulk shoot tissues using a GeneRacer ${ }^{\mathrm{TM}}$ Kit (Invitrogen) according to manufacturer's instructions in 
order to confirm that RZL03b06 represented a full-length clone.

\section{Assembly of the AtRaptor I B complementation vector}

Primers were designed to amplify the region from the end of the transcript adjacent to the AtRaptor $1 B$ locus to a site within the ORF, spanning a region from 1145 bases upstream of the transcript initiation site through to the sixth exon of the AtRaptor $1 B$ transcript. Primers used and restriction sites added are as follows: $1 \mathrm{~B}-8189 \mathrm{BglII} 5^{\prime}$ agatctgaggaaccagaagaaccc 3'; 1B+5104HindIII 5' aagcttcggcggagtaggaaaac 3'. PCR was performed using ExTaq polymerase (Takara) in a PTC-100 thermocycler on Col-0 genomic DNA with the following parameters: $96^{\circ} \mathrm{C}$ for 5 min, then 35 cycles of $94^{\circ} \mathrm{C}$ for $30 \mathrm{sec}, 60^{\circ} \mathrm{C}$ for $30 \mathrm{sec}$ and $72^{\circ} \mathrm{C}$ for $2 \mathrm{~min}$, followed by a final step of $72^{\circ} \mathrm{C}$ for 10 minutes. HindIII BglII digested fragments of the resulting PCR products were ligated into pCambia1301.

Next, the 1301B construct was digested with PmlI and PmeI. The EST containing the AtRaptor1B ORF was digested with PmeI and SmaI, and the resulting fragment was ligated into $1301 \mathrm{~B}$ to create the complementation vector 1301B:Raptor. 1301B:Raptor was transformed into Agrobacterium line GV3101, and then into Col or 1B-/plants via floral dip [32].

\section{Microscopy}

SEM fixation was performed using standard methods [33]. Bright field microscopy was performed on a Zeiss microscope, and images were collected on a BioRad Confocal System.

\section{In situ hybridization}

Digoxigenin-labelled probes were hybridized to paraffinsectioned material using previously described methods [34].

\section{In silico analysis}

Locus identifiers were submitted to the Genevestigator Arabidopsis thaliana microarray database and analysis toolbox [27] at https://www.genevestigator.ethz.ch, where they were assayed against 1434 developmental and tissuespecific Arabidopsis microarray experiment results [35-38].

\section{Authors' contributions}

GHA and MRH formulated the experiments, analyzed the data, and wrote the manuscript, GHA obtained the data for the mutant phenotypes, and BV performed the in situ hybridizations.

\section{Additional material}

\section{Additional File 1}

Alignment of Raptor homologues in plants, fungi and mammals Additional file 1 is an image of the Raptor homologue alignment. Shown are predicted protein sequences for the plant Raptor proteins AtRaptor1A and AtRaptor1B (Arabidopsis), MtRaptor (Medicago truncatula), OsRaptor (Oryza sativa), the fungal raptor homologue Mip1 (S. pombe), and mammalian Raptor. Sequences were aligned in Megalign; the image was created in Genedoc. Putative sequences for AtRaptor1 A and MtRaptor are based on genomic predictions rather than EST or cDNA sequence. Residues that are $100 \%$ conserved (either identical or biochemically similar) are shown as white text on black. More than $80 \%$ conservation is shown as white on dark grey, and more than $60 \%$ is shown as black on light grey. Click here for file

[http://www.biomedcentral.com/content/supplementary/1741-

7007-3-12-S1.ppt]

\section{Acknowledgements}

The authors gratefully acknowledge Barbara Eaglesham for assistance with scanning electron microscopy, Paulina Melechkina for the drawing in Fig. 8 and Nena Alvarez for assistance with in situ hybridization. This work was funded by a fellowship from the National Science Foundation/Department of Energy (DOE)/United States Department of Agriculture (USDA) Training Group in Molecular Mechanisms of Plant Processes to GHA, and USDA Hatch Program and DOE Energy Biosciences (DE-F602-89ER I 4030) grants to MRH. BV was supported by a Marsden award from the Royal Society of New Zealand and by the New Zealand Foundation for Research Science and Technology.

\section{References}

I. Willemsen $V$, Sheres B: Mechanisms of pattern formation in plant embryogenesis. Annu Rev Genet 2004, 38:587-6I4.

2. Zhao Y, Medrano L, Ohashi K, Fletcher JC, Yu H, Sakai H, Meyerowitz EM: HANABA TARANU Is a GATA transcription factor that regulates shoot apical meristem and flower development in Arabidopsis. Plant Cell 2004, 16:2586-600.

3. Hake S, Smith HM, Holtan H, Magnini E, Mele G, Ramirez J: The role of knox genes in plant development. Annu Rev Cell Dev Biol 2004, 20:|25-5|.

4. Poethig RS: Phase change and the regulation of developmental timing in plants. Science 2003, 301:334-6.

5. Thomas G, Sabatini D, Hall M: TOR Target of Rapamycin. In Current Topics in Microbiology and Immunology 279 New York: SpringerVerlag; 2004.

6. Jacinto $\mathrm{E}, \mathrm{Hall} \mathrm{MN}$ : Tor signalling in bugs, brain and brawn. Nat Rev Mol Cell Biol 2003, 4:I 17-26.

7. Schmelzle T, Hall MN: TOR, a central controller of cell growth. Cell 2000, 103:253-62.

8. Heitman J, Movva NR, Hall MN: Targets for cell cycle arrest by the immunosuppressant rapamycin in yeast. Science I99I, 253:905-9.

9. Kunz J, Henriquez R, Schneider U, M Deuter-Reinhard, Movva NR, Hall MN: Target of rapamycin in yeast, TOR2, is an essential phosphatidylinositol kinase homolog required for GI progression. Cell 1993, 73:585-96.

10. Barbet NC, Schneider U, Helliwell SB, Stansfield I, Tuite MF, Hall MN: TOR controls translation initiation and early $\mathrm{GI}$ progression in yeast. Mol Biol Cell 1996, 7:25-42

II. Helliwell SB, Howald I, Barbet N, Hall MN: TOR2 is part of two related signaling pathways coordinating cell growth in Saccharomyces cerevisiae. Genetics 1998, I48:99-II2.

12. Murakami M, Ichisaka T, Maeda M, Oshiro N, Hara K, Edenhofer F, H Kiyama, Yonezawa K, Yamanaka S: $\mathbf{m T O R}$ is essential for growth and proliferation in early mouse embryos and embryonic stem cells. Mol Cell Biol 2004, 24:67I0-8. 
13. Jacinto E, Loewith R, Schmidt A, Lin S, Ruegg MA, Hall A, Hall MN Mammalian TOR complex 2 controls the actin cytoskeleton and is rapamycin insensitive. Nat Cell Biol 2004, 6: I I 22-8.

14. Kim DH, Sarbassov DD, Ali SM, Latek RR, Guntur KV, ErdjumentBromage $H$, Tempst $P$, Sabatini DM: Gbetal, a positive regulator of the rapamycin-sensitive pathway required for the nutrient-sensitive interaction between raptor and $\mathbf{m T O R}$. Mol $\mathrm{Cell}$ 2003, I I:895-904

15. Kim DH, Sarbassov DD, Ali SM, King JE, Latek RR, Erdjument-Bromage $H$, Tempst $P$, Sabatini DM: mTOR interacts with raptor to form a nutrient-sensitive complex that signals to the cell growth machinery. Cell 2002, I I0:163-75.

16. Hara K, Maruki Y, Long X, Yoshino K, Oshiro N, Hidayat S, Tokunaga C, Avruch J, Yonezawa K: Raptor, a binding partner of target of rapamycin (TOR), mediates TOR action. Cell 2002, I | 0: | 77-89.

17. Loewith R, Jacinto E, Wullschleger S, Lorberg A, Crespo JL, Bonenfant D, Oppliger W, Jenoe P, Hall MN: Two TOR complexes, only one of which is rapamycin sensitive, have distinct roles in cell growth control. Mol Cell 2002, 1 0:457-68.

18. Ginalski K, Zhang H, Grishin NV: Raptor protein contains a caspase-like domain. Trends Biochem Sci 2004, 29:522-4.

19. Nojima H, Tokunaga C, Eguchi S, Oshiro N, Hidayat S, Yoshino K, Hara K, Tanaka N, Avruch J, Yonezawa K: The mammalian target of rapamycin (mTOR) partner, raptor, binds the $M T O R$ substrates p70 S6 kinase and 4E-BP I through their TOR signaling (TOS) motif. J Biol Chem 2003, 278: | 546 |-4.

20. Sarbassov DD, Ali SM, Kim DH, Guertin DA, Latek RR, ErdjumentBromage H, Tempst $P$, Sabatini DM: Rictor, a novel binding partner of $\mathrm{mTOR}$, defines a rapamycin-insensitive and raptorindependent pathway that regulates the cytoskeleton. Curr Biol 2004, I 4: 1296-302

21. Menand B, Desnos T, Nussaume L, Berger F, Bouchez D, Meyer C, Robaglia C: Expression and disruption of the Arabidopsis TOR (target of rapamycin) gene. Proc Natl Acad Sci U S A 2002 99:6422-7

22. Robaglia C, Menand B, Lei Y, Sormani R, Nicolai M, Gery C, Teoule E, Deprost D, Meyer C: Plant growth: the translational connection. Biochem Soc Trans 2004, 32:58I-4.

23. Bonnet E, Wuyts J, Rouze P, Van de Peer Y: Detection of 9 I potential conserved plant microRNAs in Arabidopsis thaliana and Oryza sativa identifies important target genes. Proc Natl Acad SciU S A 2004, 10 I: I| 151 1-6.

24. Deprost D, Truong HN, Robaglia C, Meyer C: An Arabidopsis homolog of RAPTOR/KOGI is essential for early embryo development. Biochem Biophys Res Commun 2005, 326:844-50.

25. Shinozaki-Yabana S, Watanabe Y, Yamamoto M: Novel WD-repeat protein Miplp facilitates function of the meiotic regulator Mei2p in fission yeast. Mol Cell Biol 2000, 20:1234-42.

26. Yonezawa K, Tokunaga C, Oshiro N, Yoshino K: Raptor, a binding partner of target of rapamycin. Biochem Biophys Res Commun 2004, 3 I 3:437-4I

27. Zimmermann P, Hirsch-Hoffmann M, Hennig L, Gruissem W: GENEVESTIGATOR. Arabidopsis Microarray Database and Analysis Toolbox. Plant Physiol 2004, I36:2621-32 [https://
[36 www.genevestigator.ethz.ch].

28. Rolland F, Moore B, Sheen J: Sugar sensing and signaling in plants. Plant Cell 2002, I 4(Suppl):SI85-205

29. Ward SP, Leyser O: Shoot branching. Curr Opin Plant Biol 2004, 7:73-8.

30. Shpak ED, CT Berthiaume, Hill EJ, Torii KU: Synergistic interaction of three ERECTA-family receptor-like kinases controls Arabidopsis organ growth and flower development by promoting cell proliferation. Development 2004, I 3 I: | 49 |-50 I.

31. Alonso JM, Stepanova AN, Leisse TJ, Kim CJ, Chen H, Shinn P, Stevenson DK, Zimmerman J, Barajas P, Cheuk R, Gadrinab C, Heller C Jeske A, Koesema E, Meyers CC, Parker H, Prednis L, Ansari Y, Choy N, Deen H, Geralt M, Hazari N, Hom E, Karnes M, Mulholland C, Ndubaku R, Schmidt I, Guzman P, Aguilar-Henonin L, Schmid M, Weigel D, Carter DE, Marchand T, Risseeuw E, Brogden D, Zeko A Crosby WL, Berry CC, Ecker JR: Genome-wide insertional mutagenesis of Arabidopsis thaliana. Science 2003, 30 I:653-7.

32. Clough SJ, Bent AF: Floral dip: a simplified method for Agrobacterium-mediated transformation of Arabidopsis thaliana. Plant J 1998, 1 6:735-43.
33. Jackson D, Veit B, Hake S: Expression of maize KNOTTEDI related homeobox genes in the shoot apical meristem predicts patterns of morphogenesis in the vegetative shoot. Development 1994, I 20:405-413.

34. Weigel D, Glazebrook J: Arabidopsis : A Laboratory Manual. Cold Spring Harbor Laboratory Press; 2002.

35. Edgar R, Domrachev M, Lash AE: Gene Expression Omnibus: NCBI gene expression and hybridization array data repository. Nucleic Acids Res 2002, 30:207-I0.

36. Rocca-Serra P, Brazma A, Parkinson H, Sarkans U, Shojatalab M, Contrino S, Vilo J, Abeygunawardena N, Mukherjee G, Holloway E, Kapushesky M, Kemmeren P, Lara GG, A Oezcimen, Sansone SA: ArrayExpress: a public database of gene expression data at EBI. C R Biol 2003, 326: 1075-8.

37. Parkinson H, Sarkans U, Shojatalab M, Abeygunawardena N, Contrino S, Coulson R, Farne A, Garcia Lara G, Holloway E, Kapushesky M, Lilja P, Mukherjee G, Oezcimen A, Rayner T, Rocca-Serra P, Sharma A, Sansone S, Brazma A: ArrayExpress - a public repository for microarray gene expression data at the EBI. Nucleic Acids Res 2005, 33:D553-5.

38. Craigon DJ, James N, Okyere J, Higgins J, Jotham J, May S: NASCArrays: a repository for microarray data generated by NASC's transcriptomics service. Nucleic Acids Res 2004:D575-7.
Publish with Biomed Central and every scientist can read your work free of charge

"BioMed Central will be the most significant development for disseminating the results of biomedical research in our lifetime. "

Sir Paul Nurse, Cancer Research UK

Your research papers will be:

- available free of charge to the entire biomedical community

- peer reviewed and published immediately upon acceptance

- cited in PubMed and archived on PubMed Central

- yours - you keep the copyright
BioMedcentral 